\title{
Sprachlose Medizin
}

Studenten werden vor allem visuell erzogen und später delegieren sie das Reden und Zuhören an die Pflege. Multiple-Choice-Prüfungen und computerisierte Diagnosesysteme sind Teil einer Hochtechnologie, der die Sprache abhanden kommt. Die Rechner produzieren virtuelle Realitäten, deren «harte» Daten viel mehr bedeuten als die «weichen» eines gegenseitigen Gesprächs. Wenn aber Mediziner die Wahrnehmung ihren Apparaten überlassen, eliminieren sie sich selbst. Das wäre weniger bedauerlich, wenn nicht auch die Kranken als Folge davon ihre Innenwelt ausblenden und verstummen würden. Verstehende Medizin setzt eine Sprache voraus, die natürlich und kunstvoll zugleich ist; doch wo kann diese gelernt werden? Wir alle haben oder hatten einmal ein sprachliches Ausbildungsdefizit, sind oder waren Amateure und Autodidakten, bestenfalls Naturtalente, zwischen High-Tech-Medizin auf der einen und Steinzeitkommunikation auf der anderen Seite.

In den letzten 20 Jahren hat sich in vielen Bereichen der Wissenschaft die sogenannt «narrative Wende» als neue Methode der Erkenntnis etabliert. Das Erzählen und die Analyse narrativer Texte sind ein fruchtbarer Weg, um nachzuvollziehen, wie Menschen sich selbst verstehen, wie er an seiner Biographie und Identität arbeiten kann und welche Rolle kulturelle und gesellschaftliche Prozesse dabei spielen. Gelebtes wird in narrative Muster und Motive gebracht, die ihrerseits bestimmen, was zur Erfahrung wird. Patient und Arzt «konstruieren» ihre Begegnung nach je eigenen Zielen und Herstellungslogiken. Beide erzählen eine Geschichte und folgen spezifischen Konstruktionsprinzipien. Der Patient strukturiert seine subjektive Erfahrung und der Arzt interpretiert diese aufgrund der gelernten Deutungsmuster. Die daraus entstehende «Krankengeschichte» verknüpft im idealen Fall Expertenwissen mit der subjektivexistentiellen Deutung vorgebrachter Beschwerden. Auch wenn der Arzt das Krankheitserleben und dessen Handlungsgrundlagen ignoriert, unterliegt er doch ihrer Macht und Wirkung. Was hingegen kognitiv durchschaubar ist, fördert die emotionale Resonanz und die gemeinsam erarbeiteten Handlungsspielräume und Zukunftsperspektiven. Der Heilungsprozess wird er- leichtert oder sogar erst ermöglicht. Die dazu erforderliche Sprachkompetenz entwickelt die erwünschte berufliche Sensibilität und Empathiefähigkeit.

Viele Artikel medizinischer Fachzeitschriften betonen die Vorteile der «narrativen Medizin.» In «Primary Care» [1] wurde wiederholt auf die wichtige Funktion des Erzählens hingewiesen, und auch eine längere Buchbesprechung in der «Revue Médicale Suisse» mit dem Titel «Quelle place pour l'écriture dans le milieu médical?» [2] unterstreicht die Bedeutung der Subjektivität von Therapeut und Patient als vernachlässigte Ergänzung der Apparatemedizin. Das Einfachste ist das Schwierigste, wie die Anekdote über einen Onkologen zeigt. Als er eine Patientin fragte, welchen wichtigsten Ratschlag er in einer Rede den medizinischen Studienabgängern geben könne, antwortete sie: «Sagen Sie ihnen, dass sie an meine Türe klopfen, mich begrüssen und sich verabschieden und mir in die Augen sehen sollen, wenn sie mit mir reden!»

Was die vielen Empfehlungen an Ärztinnen und Ärzte kaum je erwähnen: Wer sich nicht um seine Sprache bemüht, verkümmert nicht nur, er oder sie beraubt sich eines grössten Vergnügens. Dazu reicht ein kleiner Schritt, den der deutsche Schriftsteller Ralf Rothmann exakt umschreibt: «Ein unbeschreibliches Glück, wenn auch oft grau, eine Tortur, die Spass macht: das Schreiben. Sitzfleisch und Flausen, das ist, zunächst einmal, die Ausrüstung [...]. Plötzlich findet das Wort sich ein und macht aus dem Text ein scheinbar nie gesehenes, unerhörtes Wesen, zitternd und bis zum Zerreissen gespannt zwischen dem, was man selber wollte, und dem, was die Sprache will, ein Lebewesen, und die Welt ist momentlang am rechten Fleck. Woraufhin man den Stift in die Höhe schmeisst und vor Glück den Türpfosten küsst.» [3]

\section{Erhard Taverna}

\section{Literatur}

1 www.primarycare.ch.

2 Louis-Courvoisier M. Quelle place pour l'écriture dans le milieu médical. Rev Med Suisse. 2005.

3 Rothmann R. Stier. Roman. Frankfurt: Suhrkamp Taschenbuch 2255; 1993. 